\title{
Applications for Improving Geographic Routing Paths in Wireless Sensor Networks
}

\author{
Young-Long Chen, Wei-Jun Ding, Yung-Chi Chang, and Neng-Chung Wang
}

\begin{abstract}
This paper proposes the Geographic Multicast Fermat Point (GMFP) architecture with an Inside Relay Node (IRN). We use Fermat Point architecture to find the relay Fermat Point node to reduce the distance between received nodes. We also use the Energy-Efficient Beaconless Geographic Routing (EBGR) method to effectively find the optimal relay node position and reduce energy consumption between source nodes and Fermat Points, so that packets are forwarded for all relay nodes. Simulation results show that total energy consumption of GMFP architecture with an IRN outperforms that of GMFP architecture, thereby extending the system lifetime.
\end{abstract}

Index Terms-Fermat point, geographic routing, relay nodes, system lifetime.

\section{INTRODUCTION}

In wireless sensor networks (WSNs), the node battery power is limited. If battery power is depleted, the nodes will be unable to obtain the related functions and data [1]. Due to the geographic routing algorithm boasting low overhead and high scalability features, in this case, it is one of the most suitable routing algorithms. The geographic routing is based on a greedy forwarding algorithm [2], [3]; each node selects the nearest neighbors to forward the packet to the destination node. As an effective energy-saving routing technology, geographic routing has been identified as the most promising solution, especially in the energy-limited WSNs [4], [5].

In recent years, many research papers have proposed the using specific nodes to transmit packets in WSNs [6]-[8]; such specific nodes are called relay nodes, to forward the packets collected by the general nodes to achieve fault-tolerant load and prolong the lifetime of WSNs [9]. In this paper, Geographic Multicast Fermat Point (GMFP) architecture with Inside Relay Node (IRN) is proposed to find Fermat Points and the optimal relay node location. It can reduce the routing distance in WSNs, based on the feature of GMFP architecture. It does not need to control message flooding and maintain routing information. By relay nodes forwarding packets, the result is that the total energy consumption of all nodes is reduced and the lifetime of

Manuscript received July 15, 2013; revised September 5, 2013. This work was supported in part by the National Science Council (NSC) of Republic of China under grant No. NSC 101-2221-E-025-006.

Y. L. Chen, W. J. Ding, and Y. C. Chang are with the Department of Computer Science and Information Engineering, National Taichung University of Science and Technology, Taichung 404, Taiwan (e-mail: ylchen66@nutc.edu.tw, dream11026@gmail.com).

N. C. Wang is with the Department of Computer Science and Information Engineering, National United University, Lienda, Miaoli 360, Taiwan (e-mail: ncwang@nuu.edu.tw).
WSNs is prolonged.

The rest of this paper is organized as follows. Section II examines related work, introduces Fermat points, geographic routing algorithm, ad hoc routing, the energy mode and power-adjusted transmissions. Section III shows the proposed GMFP architecture with IRN. In Section IV the simulation investigation and comparison the performance of GMFP architecture and our proposed GMFP architecture with IRN is delineated. Finally, we summarize the paper and offer a discussion in Section V.

\section{RELATED WORK}

\section{A. Fermat Point}

In [10], for a given triangle, $\triangle A B C$, in Fig. 1. The Fermat points are defined as follows. First, check whether the three interior angles are less than or equal to 120 degrees. Next, three equilateral triangles can be drawn out: $\triangle A^{\prime} B C, \triangle B^{\prime} A C$, and $\triangle C^{\prime} A B$, based on each side as the bottom edge at $\triangle A B C$. The three equilateral triangle connect three straight lines: $\overline{A A^{\prime}}$, $\overline{B B^{\prime}}$, and $\overline{C C^{\prime}}$. The common point of intersection by the three straight lines is Fermat point $P$. The three angles $\angle A P B, \angle A P C$, and $\angle B P C$ will be equal to 120 degrees, so that the sum of the distance of $|P A|+|P B|+|P C|$ will be minimal where $|P X|$ is the distance between the Fermat point $P$ and vertex $x$.

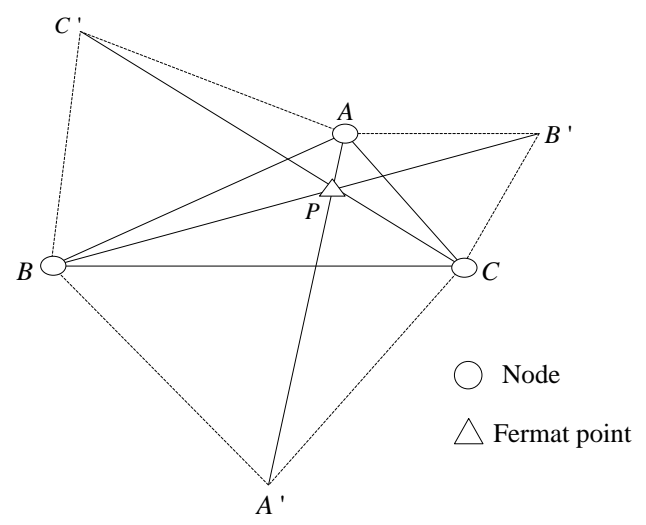

Fig. 1. Example of a Fermat point.

Theorem 1: If point $P$ is a Fermat point within the triangle, then the sum of the distances $|P A|+|P B|+|P C|$ is minimized where the three vertices: $A, B$, and $C$ of the triangle $\triangle A B C$ [10] are found, as shown in Fig. 1.

\section{B. Geographic Routing}

In recent years, varieties of different geographic routing algorithms have been proposed. Zhang and Shen [11] designed an Energy-Efficient Beaconless Geographic 
Routing (EBGR) to find the relay node to transmit packets to the sink, as shown in Fig. 2. First of all, the transmission node sends packets to the sink; the base station is not within transmission range of the node. At this time the source node calculates the optimal position of the relay node between it and the sink, and finds the relay node to forward packets to the sink.

In the past few years, Mobile Ad Hoc Networks (MANETs) [12]-[14] have become an important topic. In the MANET, the middle of the node will release small control packets; these packets left messages on each node via node forwarding. The proposed MANET reduces the total number of hops on the overall network. Many studies proposed the MANET protocol, such as Geographic Multicast Fermat Point (GMFP) architecture [12], as an effective geographical multicast protocol. In Fig. 3, the goal of GMFP architecture is to cut the distance of overall network routing. The results show that GMFP architecture provides an effective method to solve the problem of node mobility and reduce total distances between all nodes by which they can transmit and receive packets.

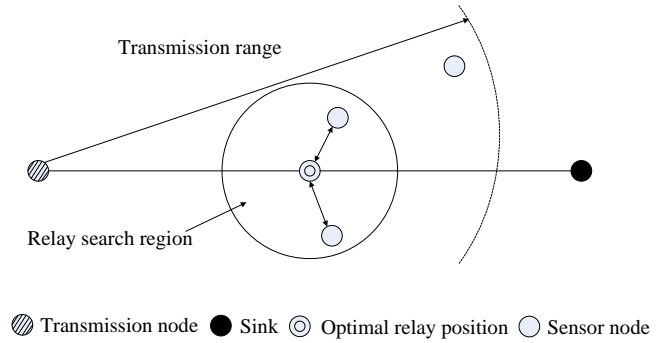

Fig. 2. EBGR architecture.

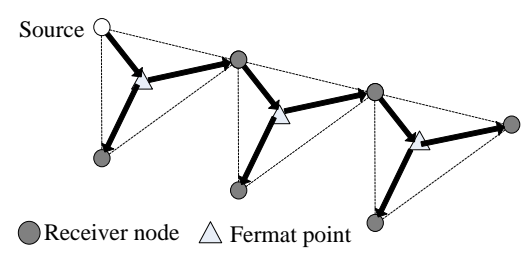

Fig. 3. GMFP architecture.

\section{Energy Model}

As Heinzelman et al. proposed [15], the first order radio model has been widely used for measuring energy consumption in WSNs [16]-[18]. The model is depicted in Fig. 4: the left side is the transmitter and right side is the receiver. Assume that the transmitter sends $l$ bits packet to the receiver through the amplifier $\varepsilon_{\text {amp }_{T_{x}}}=100 \mathrm{pJ} / \mathrm{bit} / \mathrm{m}^{2}$ over distance $d$. Then, energy consumption of the transmitter is given by:

$$
E_{T x}(l, d)=E_{\text {elec } T ⿱ 乛 龰_{T x}} \times l+\varepsilon_{\text {amp }_{T x}} \times l \times d^{\alpha}
$$

where $E_{\text {ele }_{T_{x}}}=50 \mathrm{~nJ} / \mathrm{bit}$ is energy consumption of the transmitter, and $\alpha$ is path loss coefficient. According to different transmission distances, the decay rate of packets is not the same for $\varepsilon_{a m p_{T x}}$ and $\alpha$. The path loss coefficient is due to the signal strength attenuation with increasing transmission distance. The energy consumption of receiver is shown as:

$$
E_{R x}(l)=E_{\text {elec }_{R x}} \times l
$$

where $E_{\text {elec }_{R x}}$ is energy consumption of the receiver.

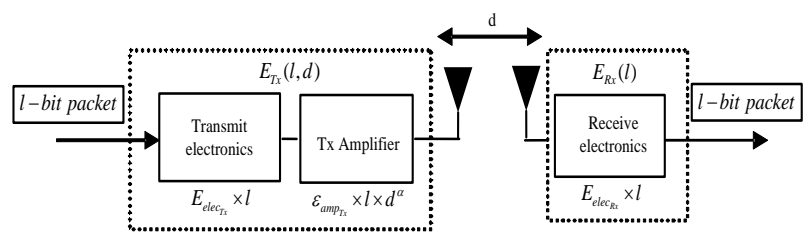

Fig. 4. First order radio model.

\section{Power-Adjusted Transmissions}

Stojmenovic and Lin [5] proposed the power-adjusted transmissions based on the First Order Radio Model. Assuming the 1 bit packet energy required for transmitter and receiver over distance $d$, it is given by:

$$
e(d)=\left(\varepsilon_{\text {amp Tx }_{T x}} \times d^{\alpha}\right)+\left(E_{\text {elec }_{T x}}+E_{\text {elec } R x_{R x}}\right)
$$

where $E_{\text {ele }_{T x}}$ is the energy consumption of transmitter, $E_{\text {elec }_{R x}}$ is energy consumption of receiver and $\alpha$ is the path loss coefficient.

The characteristics of power-adjusted transmissions approach are as follows. For a given source node $s$ and a destination node $u, d$ is the distance between node $s$ and node $u$, and $e(d)$ is the total energy consumed by transmitting 1 bit packet from $s$ to $u$. The transmissions threshold [18] is defined as follows:

$$
d_{\text {threshold }}=\sqrt[\alpha]{\frac{E_{\text {elec }_{T x}}+E_{\text {elec }_{R x}}}{\varepsilon_{\text {amp }_{T x}} \times\left(1-2^{1-\alpha}\right)}}
$$

If $d$ is smaller than $d_{\text {threshold }}$, then the direct transmission is the most energy-efficient way to transmit packets from node $s$ to node $u$. If $d$ is larger than $d_{\text {threshold }}$, then the packets are transmitted by relay nodes; this can save more energy than the packets directly transmitted without relay nodes.

\section{Geographic Multicast Fermat Point (GMFP) ARCHITECTURE WITH INSIDE RELAY NODE (IRN)}

In this paper, we proposed GMFP architecture with IRN. To find the optimal path, the total energy consumption of all nodes is the minimum and prolongs the lifetime of the wireless sensor network. GMFP architecture with IRN is divided into three phases: $A$. initial topology setup phase; $B$. the Fermat points phase; $C$. inside relay nodes selection phase.

\section{A. Initial Topology Setup Phase}

In the first step, each node links to other nodes by multiple broadcasts, so that the source node can send packets to any receiving node. Then, the source node based on Gabriel graphic (GG) architecture [19], establishes a straight line 
between two nodes and draws a circle via a diameter's straight line. The distance between two nodes is the closest. After we use the GG architecture to establish the network topology, there are several of triangles of the circulation circuit, as shown in Fig. 5.

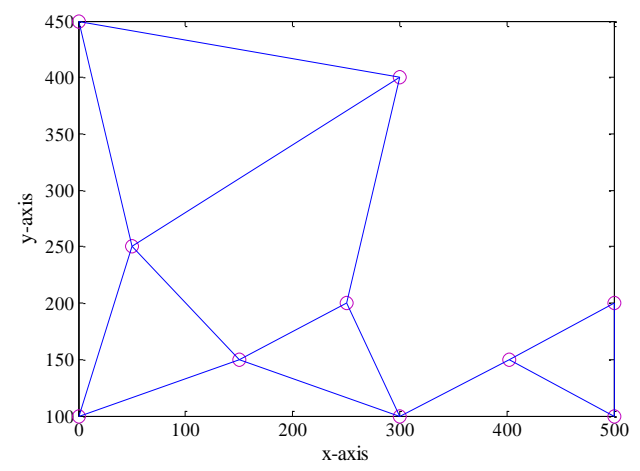

Fig. 5. The network topology based on GG.

\section{B. The Fermat Points Phase}

The rules of GG between nodes will produce some circulation path (e.g., triangles). According to the theorem of Fermat points, these triangles may contain a Fermat point. In geometry, for a given a triangle $\triangle A B C$, Fermat point $P$ is a point located within the triangle [10]. The sum of the distances between the Fermat point $P$ and the three vertices: $A, B$, and $C$ of the triangle $\triangle A B C$ are shown in Fig. 1. Fermat points have been proved in [10]; the distance of Fermat point $P$ to the three vertices is the minimum one. Therefore, we will explore the establishment of the network topology triangle in GG at this phase, and check these triangles to verify if a Fermat point exists.

In Fig. 6, the Fermat point algorithm is used to find the Fermat point (red triangle) in a WSN; the entire backbone network will be constructed. The Fermat point is calculated according to the literature [12], as the node might not be in the position of Fermat point. Therefore, we will select the node nearest the Fermat point as the actual Fermat point. Next, we will look for relay nodes, to further reduce the energy consumption of nodes.

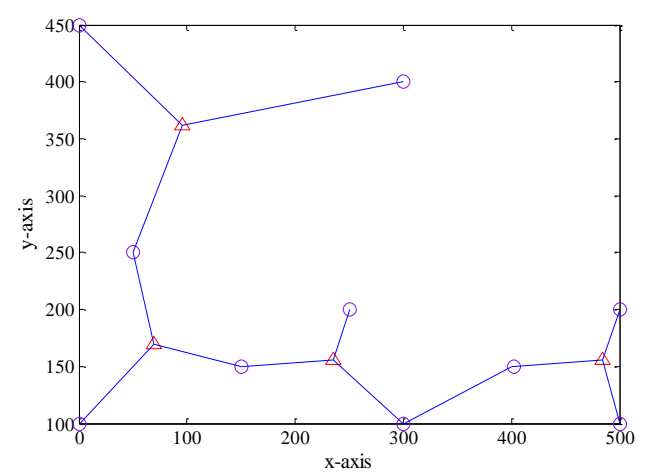

Fig. 6. The Fermat point backbone network is constructed.

\section{Inside Relay Nodes Selection Phase}

In Fig. 7, it is assumed that source node $v$ transmits packets to Fermat point $f$; we judge whether to select the relay nodes from the inside Fermat point through relay nodes, to forward packets to other Fermat points and other nodes.

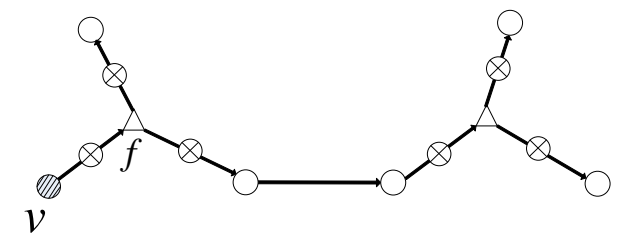

Source node $\bigcirc$ Node $\triangle$ Fermat point $\otimes$ Inside relay node

Fig. 7. Select relay node from the inside Fermat point.

According to Stojmenovic and Lin's scheme [5], we calculate the distance $d_{v f}$ between the source node $v$ and the Fermat point $f$. If the distance $d_{v f}$ is smaller than $d_{\text {threshold }}$ in (4), then the source node $v$ will directly transmit packets to the Fermat point $f$. Packets forwarded by relay nodes consume more total energy than packets which are directly transmitted.

Besides, if the distance $d_{v f}$ is larger than $d_{\text {threshold }}$ in (4), we transmit packets by relay nodes to reduce energy consumption more than when packets are directly transmitted. Therefore, the source node $v$ will select relay nodes between Fermat point $f$, so that forwarded packets can save energy, as follows:

We find relay node by [11] which forwards packets to the Fermat point $f$ between the source node $v$ and Fermat point $f$. First of all, let $\left(x_{v}, y_{v}\right)$ and $\left(x_{f}, y_{f}\right)$ represent the coordinate of the source node $v$ and the Fermat point $f$, respectively. Fig. 8 shows the relay node search range is $R_{i}$, $i$ is defined as the relay node search position in inside Fermat point and $r_{i}$ is defined as relay node search radius. $R_{i}$ must satisfy:

$$
R_{i} \leq \frac{d_{v f}}{2}
$$

And $\left(x_{i}, y_{i}\right)$ represents the location of relay node search position $i$. If the coordinates of the source node $v$ and the Fermat point $f$ are known, then $\left(x_{i}, y_{i}\right)$ can be computed as follows:

$$
\left\{\begin{array}{l}
x_{i}=x_{v}-\frac{r_{i}}{d_{v f}}\left(x_{v}-x_{f}\right) \\
y_{i}=y_{v}-\frac{r_{i}}{d_{v f}}\left(y_{v}-y_{f}\right)
\end{array}\right.
$$

When node $v$ has a packet to be transmitted, it will broadcast a message, wherein the message contains the $i$, $R_{i}$ and $r_{i}$, to determine the next relay node. Assume that any node $u$ receives a message from node $v$; it checks whether it is in the relay node search position. If it is not, node $u$ will discard this message. Otherwise, node $u$ will select a node nearest $i$ within the relay node search range $R_{i}$ to act as the relay node. 


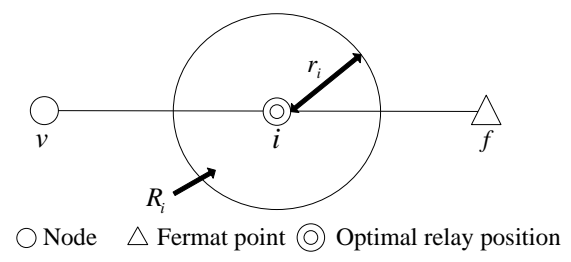

Fig. 8. The range of relay node search for inside Fermat point.

When node $v$ has a packet to be transmitted, it broadcasts a message from the relay node search position $i$. If the broadcast message is firstly received by node $u$, it indicates that it is nearest the relay node location $i$. Node $\mathrm{u}$ will return a broadcast message to node $v$, let the relay node by node $u$ to serve as. Then node $v$ will begin transmitting packets to the relay node $u$, and node $u$ will forward packets to Fermat point $f$, as shown in Fig. 9. Finding the transmission path allows the individual nodes to achieve the minimum energy consumption, to extend the lifetime of the sensor network.

\section{Simulation Results}

To assess the performance of our proposed GMFP architecture with IRN, we use Matlab to perform the simulation. In this simulated environment, we compared GMFP architecture [12], energy consumption for different numbers of nodes, total squared Euclidean distance, and the energy consumption in different node mobility rate. In all of the simulation parameters, the sensor area size is $500 \mathrm{~m} \times 500 \mathrm{~m}$ The energy module parameters are set as follows: path loss coefficient $\alpha=2$, the sender and receiver energy consumption needed for transmitting packets is $50 \mathrm{~nJ} / \mathrm{bit}$, energy consumed for amplifier is $100 \mathrm{pJ} / \mathrm{bit} / \mathrm{m}^{2}$, and the number of packets is $l=4000 \mathrm{bits}$. All sensing nodes maximum sensing range is set at $R=80 \mathrm{~m}$, and the sensing radius is $r=40 \mathrm{~m}$. There simulation sensing nodes number 100 and 300 randomly deployed in the sensor area size $500 \mathrm{~m} \times 500 \mathrm{~m}$. And 9 receive nodes be chosen. Node mobility rate is set as follows: $0 \mathrm{~m} / \mathrm{s}, 5 \mathrm{~m} / \mathrm{s}, 10 \mathrm{~m} / \mathrm{s}, 15 \mathrm{~m} / \mathrm{s}$ and $20 \mathrm{~m} / \mathrm{s}$; then the range of the nodes moving direction is $[0,2 \pi]$. Each time of movement has a $10 \mathrm{~s}$ interval, and the new direction will be re-selected at the interval between the ends of each mobility rate.

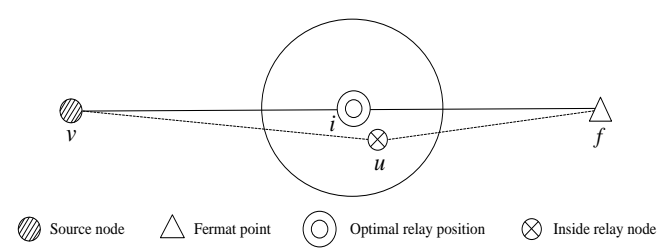

Fig. 9. Packets by relay nodes transmit.

In Figs. 10-12, the simulation results show that we compared three different performances in the 100 number of nodes and the moving speed of the nodes is $10 \mathrm{~m} / \mathrm{s}$.

The simulation results in Fig. 10 show 10 kinds of different network topologies. In the six topology GMFP architectures, energy consumption is about $0.40432 \mathrm{~J}$; our proposed GMFP architecture with an IRN for energy consumption is about
$0.33832 \mathrm{~J}$. It can be seen that the energy consumption has significantly decreased in 10 kinds of different network topologies.

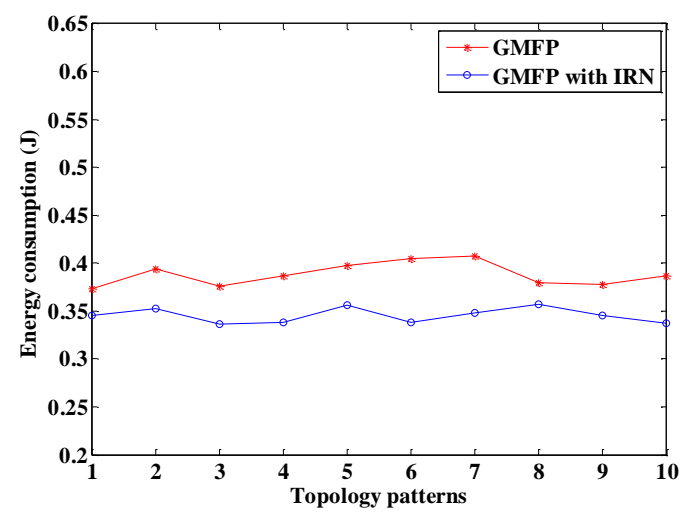

Fig. 10. Energy consumption of nodes in the different topology patterns with 100 nodes.

In Fig. 11, the total squared Euclidean distance has clearly decreased compared to the GMFP architecture. In the first topology GMFP architectures, energy consumption is about $960 \mathrm{~m}^{2}$. We proposed GMFP architecture with IRN for total squared Euclidean distance is about $790 \mathrm{~m}^{2}$.

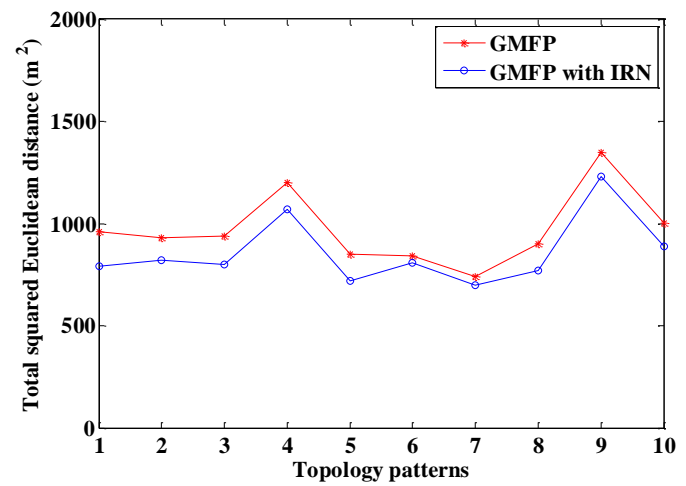

Fig. 11. Total squared Euclidean distance in different topology patterns with 100 nodes.

In Fig. 12, we compare the energy consumption in the different nodes' mobility rates. Energy consumption of the GMFP architecture is about $0.39754 \mathrm{~J}$ in the nodes mobility rate of $5 \mathrm{~m} / \mathrm{s}$. In our proposed GMFP architecture with IRN, energy consumption is about $0.34499 \mathrm{~J}$ in the node mobility rate of $5 \mathrm{~m} / \mathrm{s}$.

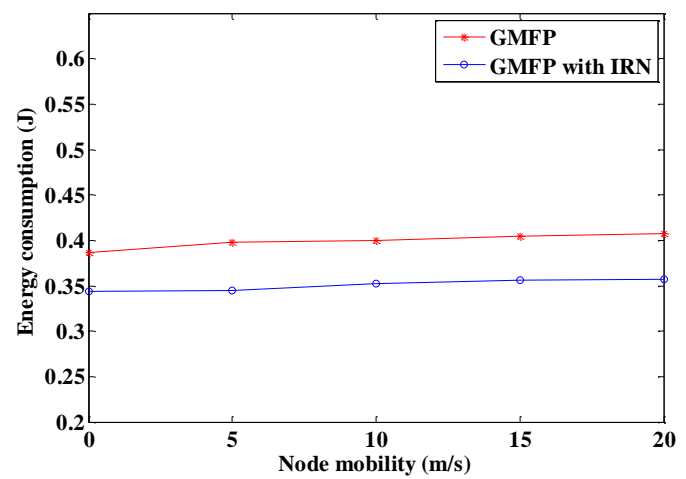

Fig. 12. Energy consumption in different mobility rate with 100 nodes.

Figs. 10-12 show that the performance of our proposed GMFP architecture with IRN outperforms that of the GMFP 
architecture in terms of energy consumption and the total squared Euclidean distance.

\section{CONCLUSION}

In this paper, we combined the Fermat point with the relay node selection method, and then we proposed GMFP architecture with IRN. We utilized the characteristics of the Fermat point to reduce the route distance, and the transmission of packets through geographic routing to forward packets does not require complex control of messages or maintaining complex neighbor information. It is simple and does not require complicated calculation. Simulation results show that in our GMFP architecture with IRN compared with GMFP architecture in 100 nodes, the energy consumption, total squared Euclidean distance, and energy consumption in the different mobility rate significantly improve.

\section{REFERENCES}

[1] Z. Cheng, M. Perillo, and W. B. Heinzelman, "General network lifetime and cost models for evaluating sensor network deployment strategies," IEEE Trans. Mobile Computing, vol. 7, no. 4, pp. 484-497, Apr. 2008.

[2] D. Chen and P. Varshney, "A survey of void handling techniques for geographic routing in wireless networks," IEEE Communications Surveys \& Tutorials, vol. 9, no. 1, pp. 50-67, May 2007.

[3] G. W. Denardin, C. H. Barriquello, A. Campos, and R. N. Prado, "A geographic routing hybrid approach for void resolution in wireless sensor networks," The Journal of Systems and Software, vol. 84, no. 10 , pp. 1577-1590, Oct. 2011.

[4] K. Akkaya and M. Younis, "A survey on routing protocols for wireless sensor networks," Ad Hoc Networks, vol. 3, no. 3, pp. 325-349, May 2005.

[5] I. Stojmenovic and X. Lin, "Power-aware localized routing in wireless networks," IEEE Trans. Parallel Distrib. Syst., vol. 12, no. 11, pp. 1122-1133, Nov. 2001.

[6] A. Efrat, S. P. Fekete, P. R. Gaddehosur, J. S. B. Mitchell, V. Polishchuk, and J. Suomela, "Improved approximation algorithms for relay placement," in Proc. the 16th European Symposium on Algorithms, Karlsruhe, Germany, Sept. 2008, pp. 356-367.

[7] M. Younis and K. Akkaya, "Strategies and techniques for node placement in wireless sensor networks: a survey," Ad Hoc Networks, vol. 6, no. 4, pp. 621-655, June 2008.

[8] S. Lee and M. Younis, "Optimized relay node placement for connecting disjoint wireless sensor networks," Computer Networks, vol. 56, no. 12 pp. 2788-2804, Aug. 2012.

[9] B. Ataul, W. Shamsul, J. Arunita, and B. Subir, "A genetic algorithm based approach for energy efficient routing in two-tiered sensor networks," Ad Hoc Networks, vol. 7, no. 4, pp. 665-676, June 2009.

[10] D. Pedoe, Geometry, a Comprehensive Course, New York: Dover Publications, 1988.

[11] H. Zhang and H. Shen, "Energy-Efficient Beaconless Geographic Routing in Wireless Sensor Networks," IEEE Trans. Parallel Distrib. Syst., vol. 21, no. 6, pp. 881-896, June 2010.

[12] K. F. Ssu, C. H. Yang, C. H. Chou, and A. K. Yang, "Improving routing distance for geographic multicast with Fermat points in mobile ad hoc networks," Computer Networks, vol. 53, no. 15, pp. 2663-2673, Oct. 2009.

[13] C. C. Shen and C. Jaikaeo, "Ad hoc multicast routing algorithm with swarm intelligence," Mobile Networks and Applications, vol. 10, no. 1, pp. 47-59, Feb. 2005.

[14] M. Mauve, H. Fubler, J. Widmer, and T. Lang, "Position-based multicast routing for mobile ad-hoc networks," ACM Mobile Computing and Communications Review, vol. 7, no. 3, pp. 53-55, July 2003.

[15] W. R. Heinzelman, A. Chandrakasan, and H. Balakrishnan, "Energy-efficient communication protocol for wireless microsensor networks," in Proc. the 33rd Annual Hawaii International Conference on System Sciences, Hawaii, USA, Jan. 2000, pp. 4-7.
[16] M. Bhardwaj, T. Garnett, and A. P. Chandrakasan, "Upper bounds on the lifetime of sensor networks," in Proc. IEEE International Conference on Communications, Helsinki, Finland, vol. 3, June 2001, pp.785-790.

[17] Q. Li, J. Aslam, and D. Rus, "Distributed energy-conserving routing protocols for sensor network," in Proc. the 36rd Hawaii International Conference System Sciences, Hawaii, USA, pp. 4, Jan. 2003.

[18] T. Melodia, D. Pompili, and I. F. Akyildiz, "Optimal local topology knowledge for energy efficient geographical routing in sensor networks," in Proc. Twenty-third Annual Joint Conference of the IEEE Computer and Communications Societies, Hong Kong, PR China, vol. 3, Mar. 2004, pp. 1705-1716.

[19] B. Karp and H. T. Kung, "GPSR: greedy perimeter stateless routing for wireless networks," in Proc. the 6th Annual International Conference on Mobile Computing and Networking, Boston, USA, Aug. 2000, pp. 253-254.

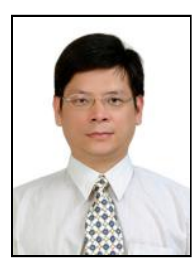

Young-Long Chen received the B.S. degree in automatic control engineering from Feng Chia University, Tai-Chung, Taiwan, in 1988, the M.S. degree in engineering science from National Cheng Kung University, Tainan, Taiwan, in 1995 and the Ph.D. degree in electrical engineering from National Chung Cheng University, Chia-Yi, Taiwan, in 2007. From 1995 to 1999 he worked for Formosa Petrochemical Corporation as a Design Engineer. From 1999 to 2007, he was a Lecturer with the Department of Electrical Engineering, Chienkuo Technology University, Taiwan. From 2007 to 2009, he was an Associate Professor with the Department of Electrical Engineering, Chienkuo Technology University, Taiwan. Since 2009, he has been with the Department of Computer Science and Information Engineering, National Taichung University of Science and Technology, Taiwan, where he is currently a Professor. His research interests include wireless and mobile communications and networks, wireless sensor networks, information security, digital signal processing, fuzzy neural networks and embedded systems. He is a member of the IEEE.

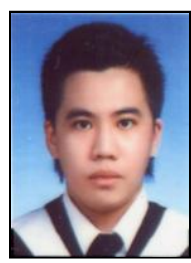

Wei-Jun Ding received the B.S. degree in Department of Computer Science and Information Engineering from National Taichung Institute of Technology, Taichung, Taiwan, in 2010. The M. S. degree is received in department of computer science and information engineering from National Taichung University of Science and Technology, Taichung, Taiwan, in 2012. His research interests in wireless sensor networks, mobile communications, and embedded systems.

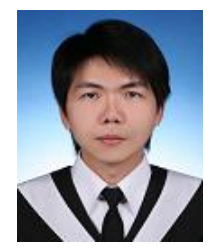

Yung-Chi Chang received the B.S. degree in department of computer science and information engineering from National Taichung University of Science and Technology, Taichung, Taiwan, in 2011. He is currently a Student in department of computer science and information engineering from National Taichung University of Science and Technology, Taichung, Taiwan. His research interests include wireless sensor networks, mobile communications, and embedded systems.

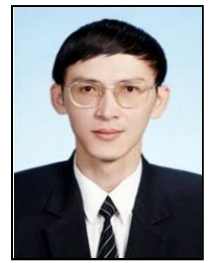

Neng-Chung Wang received the B.S. degree in Information and Computer Engineering from Chung Yuan Christian University, Taiwan, in June 1990, and the M.S. and Ph.D. degrees in Computer Science and Information Engineering from National Cheng Kung University, Taiwan, in June 1998 and June 2002, respectively. He joined the faculty ofthe Department of Computer Science and Information Engineering, Chaoyang University of Technology, Taiwan, as an assistant Professor in August 2002. From August 2006 to July 2007, he was an Assistant Professor at the Department of Computer Science and Information Engineering, National United University, Taiwan. Since August 2011, he has become a Professor at the Department of Computer Science and Information Engineering, National United University, Taiwan. His current research interests include wireless and mobile networks, wireless communication protocols, mobile computing, and parallel and distributed computing. He is a member of the IEEE, IEEE Communications Society, and Phi Tau Phi Society. 